\title{
HOW FIRMS CREATE VALUE THROUGH CORPORATE SOCIAL PERFORMANCE: A META-ANALYSIS
}

\author{
PUSHPIKA VISHWANATHAN \\ Amsterdam Business School, University of Amsterdam \\ Plantage muidergracht 12, 1018 TV Amsterdam, The Netherlands \\ P.Vishwanathan@uva.nl \\ PATRICIO DURAN \\ Business School, Universidad Adolfo Ibáñez, Chile
}

The key focus of Corporate Social Responsibility (CSR) research has historically been the relationship between Corporate Social Performance (CSP) and Corporate Financial Performance (CFP). Given the normative underpinnings of CSR (Donaldson and Preston, 1995; Wood, 1991), and the field's historical positioning as juxtaposing CSR next to the classical economic conception of the firm (Friedman, 1970), it is not surprising that the empirical question of whether CSP harms or helps CFP has triggered a lot of attention. Current research, however, has shifted the focus from studying whether a relationship exists between CSP and CFP, to uncovering how CSP and CFP may be connected (Wang, et al., 2016). Numerous moderating and mediating factors impacting this core relationship have been documented, including variables capturing effects of the institutional context (Julian and Ofori-Dankwa, 2013), the type of CSP activity (Barnett and Salomon, 2006), the extent of market competition (Flammer, 2015), legitimacy (Bansal and Clelland, 2004), long-term orientation (Wang and Bansal, 2012), intangible asset development (Surroca et al., 2010), cost of capital reductions (Cheng, Ioannou, and Serafeim, 2014), increased customer loyalty (Sen and Bhattacharya, 2001), and so on.

In addition to investigating these finer-grained mechanisms, recent CSP research has also drawn rather eclectically on different theoretical perspectives that are frequently invoked in the strategic management literature, such as the resource-based view (RBV: Barney, 1991; Hart, 1995; Surroca et al., 2010; Wernerfelt, 1984), transaction cost theory (Cheng et al., 2014; Jones, 1995; Williamson, 1975, 1985), and institutional theory (Bansal and Clelland, 2004; Campbell, 2007; DiMaggio and Powell, 1983; Meyer and Rowan, 1977);). Because these multi-theoretical underpinnings derive from different base disciplines and are often not wholly commensurable, they have left the field of CSP research in a theoretically eclectic and fragmented state. While we concur with the observation that CSP is a multidisciplinary research field (Lockett et al., 2006; Parmar et al., 2010) and acknowledge that the theoretical pluralism that has dominated the field to date has uncovered many valuable insights, we also observe that after four decades of theoretically fragmented research there remains a high degree of ambiguity with regard to the key relationships that connect CSP to CFP.

The main aim of this study is to create more clarity regarding the CSP-CFP relationship by asking: What are theoretically the key mediating pathways through which CSP may translate into higher CFP? We answer this question by developing and empirically testing an integrative theoretical framework that identifies a limited set of mediating pathways through which CSP creates value for the both the firm's stakeholders and the firm itself. Although our theory draws on insights from other theoretical perspectives, including the ones described above, it is grounded in a CSR (Carroll, 1979, 1999; Garriga and Melé, 2004; Matten and Moon, 2008; Wood, 1991) and stakeholder theory (Clarkson, 1995; Freeman, 2010; Freeman et al., 2004; Rowley, 1997) derived view of how firms identify and cooperate with stakeholders in ways that will ultimately benefit themselves as well. We test our theoretical framework by means of a meta-analysis of 344 prior studies on the 
relationship between CSP and CFP. A meta-analysis rather than a primary study is most appropriate to answer our research question, because it allows us to reorganize and reinterpret the vast empirical evidence accumulated to date. We test the four hypothesized mediating pathways with the help of meta-analytical structural equation modeling (MASEM: Aguinis et al., 2011; Bergh et al., 2016), a meta-analytical technique that is increasingly being used by strategic management scholars (e.g., Carney et al., 2011; Drees and Heugens, 2013; Duran et al., 2016; Karna et al., 2016; Van Essen et al., 2015);).

By proposing a theoretical framework that integrates four mediating pathways through which firms create value from their investments in CSP, we aim to make two key contributions to the literature. First, by rooting our framework firmly in the CSR and instrumental stakeholder theory literatures, we offer clarity and structure to the CSP-CFP debate. By coalescing a diverse and fragmented body of research around a select few perspectives, we provide a parsimonious and coherent account of the key mediating pathways through which CSP and CFP are connected. Moreover, we test our theory on the most comprehensive dataset of primary studies on the CSP-CFP relationship to date, and find strong empirical support for our predictions. Second, our theory contributes to CSP research more generally. Due to the theoretically eclectic nature of this research field, the field has been limited in its ability to establish a dominant paradigm and 'claim substantive/systematic distinctiveness' from other fields (Lockett et al., 2006: 117). Our model advances CSP as a research field by taking the CSP-CFP relationship as a central focus of this literature, and by developing a theoretical framework capturing the multiple pathways mediating this relationship. Because our framework is singularly grounded in the complementary CSR and stakeholder perspectives (Clarkson, 1995; McWilliams and Siegel, 2001) rather than in multiple incommensurable or diffracted theoretical perspectives, it has the potential to provide a more unified theoretical understanding and organization of the field.

\section{THEORY AND HYPOTHESES}

As others have already provided excellent narrative reviews of the evolution of the CSR and stakeholder concepts (e.g., Aguinis and Glavas, 2012; Laplume et al., 2008; Lockett et al., 2006; Parmar et al., 2010), we will not attempt to do the same here. Instead, we will draw on the foundational tenets of the CSR and (instrumental) stakeholder theory literatures, in order to construe an integrative theoretical framework capturing the value creation pathways involving CSP (cf. Clarkson, 1995; McWilliams and Siegel, 2001).

Although CSR and stakeholder theory have often been used interchangeably, there are subtle but important theoretical differences between the two theoretical perspectives. The most important one lies in the different points of view that these two theories take up. In his foundational contribution to the CSR literature, Carroll (1979: 500) stated that 'The social responsibility of business encompasses the economic, legal, ethical, and discretionary expectations that society has of organizations at a given point in time.' The CSR literature thereafter adopted a distinctively societal point of view, as it addressed the question of what society considers the responsibilities of businesses to be (Carroll, 1999; Matten and Moon, 2008; Wood, 1991). As a result, the CSR literature generally adopts a highly inclusive understanding of what the social responsibilities of business entail and of who might be the designated beneficiaries of these.

Stakeholder theory, on the other hand, was developed primarily because the traditional 'production view of the firm' (Freeman, 2010: 5) did not provide sufficient understanding of how changes in the external environment would affect firms, and how firms should respond to such changes. The guiding idea behind the stakeholder approach, Freeman argued, is that it would help executives to manage adaptation to their firms' external 
environments more effectively. Stakeholder theory is thus developed from an explicitly managerial point of view, and also puts a much stronger emphasis on questions of strategic management as explicitly articulated by Freeman: 'My emphasis throughout will be on strategic management, that is, how can the stakeholder concept be used to enrich our understanding of how organizations do, and should, set and implement strategy' (p: 47).

In spite of these differences, the CSR and stakeholder perspectives complement each other and jointly inform our understanding of the mediating pathways through which CSP may affect CFP. While the CSR literature presents a broad, societal perspective on understanding and explaining firm actions, stakeholder theory offers a more focused and managerial perspective to ensure that the CSP decisions that are taken are also valueenhancing for the firm. The societal perspective adopted by the CSR literature is premised on the notion that 'business exists at the pleasure of society' (Wartick and Cochran, 1985: 759), and thus, its behavior must fall within the expectations set by society. Without entering the normative debate about whether firms live up to these expectations because of felt moral obligations or because of enlightened self-interest (Donaldson and Preston, 1995), we can expect that those firms that do live up to these expectations will be perceived as being more legitimate and favorable by society. This positive societal stance toward the firm manifests itself in the every-day reality of managers in the form of more cooperative primary and secondary stakeholder relationships. We propose that when stakeholders are more cooperative, they will help firms and their managers to create more value, both directly and indirectly. They do so directly through their willingness to make existing or future relationships with the firm more productive. They also facilitate value creation indirectly, because their willingness to share valuable information with the firm permits managers to better identify opportunities and threats and develop adequate strategic responses to them.

Building on this foundational logic, we distinguish four mediating pathways through which managers can use CSP to create value for their firms. First, we hypothesize that CSP will increase stakeholder endorsement of the firm, which will make relationships that firms have with their stakeholders either more cost-efficient or more revenue-generating. Second, firms that engage in CSP will have a stronger reputation, enabling them to attract prospective stakeholders while also deepening relationships with existing stakeholders. Third, CSP will strengthen the firm's innovative capacity and thereby its ability to identify and seize novel opportunities. And fourth, CSP will reduce the firm's risk exposure, due to its improved ability to avoid stakeholder threats or minimize their impact. We support these hypotheses with an elaborate review of existing empirical and theoretical work, but due to space limitations, we only summarize the hypotheses here:

Hypothesis 1: CSP enables firms to increase stakeholder endorsement, which in turn will positively affect CFP.

Hypothesis 2: CSP enables firms to increase their reputation, which in turn will positively affect CFP.

Hypothesis 3: CSP enables firms to increase their innovative capacity, which in turn will positively affect CFP.

Hypothesis 4: CSP enables firms to create value by reducing firm specific risk, which in turn is negatively related to CFP.

\section{METHODS}


To identify the population of studies on CSP, we used five complementary search strategies: we read existing review articles and meta-analytical studies on the CSP-CFP relationship, we examined six electronic databases using relevant search terms, we manually searched 15 journals in the fields of management and finance, we corresponded via email with all researchers who had written empirical papers on CSP with missing effect-size information, asking them for a correlation table and any studies we could not retrieve by other means, and finally, we backward-traced all references reported in the identified articles and forward-traced all articles that cited the original articles via Google Scholar. These five search strategies yielded a final sample of 344 primary studies. Of these, 296 were published journal articles and 48 were working papers at the time of our analysis.

After reading the articles, we developed a coding protocol (Lipsey and Wilson, 2001) for extracting data on relevant variables. For testing the hypotheses, we collected Pearson product-moment correlations and sample size information for all the variables in our study, including effect sizes for the interrelationships between all dependent, independent, mediator, and control variables used in our analyses.

We used Hunter and Schmidt (2004) meta-analysis (HSMA) to compute the metaanalytic mean effect size for the relationship between CSP and CFP. We also performed a variety of sub-group analyses to assess whether meta-analytic mean effect size was sensitive to: (a) the source of the CSP data (survey measure, archival measures, or third-party evaluations); (b) the stakeholder group targeted with the CSP activities (employees, customers, suppliers, shareholders, community, natural environment, or mixed stakeholders); (c) the CFP measures used (accounting- or market-based); and (d) the industry context in which the measurement took place (manufacturing, financial services, non-financial services, or mixed industries).

We used meta-analytical structural equation modeling (MASEM; Aguinis et al., 2011; Bergh et al., 2016; Carney et al., 2011) to test our hypotheses. The MASEM procedure combines the techniques of structural equation modeling with those of meta-analysis (Cheung and Chan, 2005). The technique is ideally suited to test our hypotheses, because it allows us to analyze: (a) the direct effect of CSP on CFP, (b) the effect of CSP on stakeholder endorsement, firm reputation, innovative capacity, and firm risk, and (c) the consequences of these mediating variables on CFP (Baron and Kenny, 1986).

We conducted our MASEM modeling with the help of a two-stage procedure (Bergh et al., 2016). First, a series of separate HSMA analyses are conducted to construct a metaanalytic correlation table specifying the mean correlations between all independent, mediator, dependent, and control variables. To deal with sample size differences across the correlation coefficients included in our matrix, we based our analysis on the harmonic mean sample size (Geyskens et al., 2006). In the second stage, the meta-analytic correlation matrix is treated as the observed correlation matrix, and subjected to regular maximum likelihood structural equation modeling routines to test the hypothesized relationships (Cheung and Chan, 2005).

\section{RESULTS}

According to our results, the meta-analytic corrected mean effect size or the focal relationship is 0.07 . This effect size is somewhat smaller but comparable in terms of magnitude to those reported in prior meta-analyses (Margolis et al., 2007: 0.13; Orlitzky et al., 2003: 0.18).

Sub-group HSMA analyses reveal that the heterogeneity in study findings is at least partially driven by methodological artifacts. Our results indicate that when CSR is measured using survey data, the effect size is significantly higher than when CSR is measured using archival data or third-party rater data. Like Orlitzky and associates (2003), we also found 
CSP to be (somewhat) more strongly linked to accounting-based measures of performance than to market-based measures. We found limited evidence for moderation by industry context as well as stakeholder group.

Figure 1 illustrates Hypotheses 1 through 4. To investigate whether a direct and significant association between CSP and CFP exists, or whether this relationship is fully mediated by our hypothesized mediation variables, we estimated two nested models, allowing us to trace changes in the coefficient for the direct relationship. We commenced with a simple model examining the direct relationship between CSP and CFP while controlling for prior firm performance, firm size, firm leverage, and capital intensity. To test our hypotheses we subsequently ran a more elaborate model including (1) firm reputation, (2) stakeholder endorsement, (3) firm risk, and (4) innovative capacity as mediating variables. We controlled for prior CFP, firm size, firm leverage and capital intensity for each mediating variable. The harmonic mean for this elaborate model was 2,798 observations. The model fits the data well $\left(\chi^{2}=119.52 ; \mathrm{RMSR}=0.024 ; \mathrm{GFI}=0.99\right)$.

Insert Figure 1 about here

We find support for Hypothesis 1. CSP is positively related to stakeholder endorsement $(\beta=0.04, p<0.05)$, and stakeholder endorsement is positively related to CFP $(\beta$ $=0.05, p<0.05)$. Hypothesis 2 likewise receives empirical support. CSP is positively related to firm reputation $(\beta=0.10, p<0.05)$, and firm reputation is positively related to CFP ( $\beta=$ $0.05, p<0.01)$. Hypothesis 3 is also supported. CSP is positively related to innovative capacity $(\beta=0.07, p<0.05)$, and innovative capacity is positively related to CFP $(\beta=0.05, p$ $<0.05)$. Finally, we found support for Hypothesis 4 . CSP is negatively related to firm risk $(\beta$ $=-0.04, p<0.05)$, and firm risk is negatively related to CFP $(\beta=-0.09, p<0.05)$. Jointly, these four variables partially mediate the relationship between CSP and CFP, as the focal effect remains significant $(\beta=0.07, p<0.05)$ upon their inclusion. Formal tests for statistical mediation confirm that relationship between CSP and CFP is channeled through the four hypothesized mediation paths (Sobel: $z=5.89, p<0.001$; Aroian: $z=5.83, p<0.001$; Goodman: $z=5.95, p<0.001$; cf. MacKinnon et al., 1995).

We performed several additional analyses to address a number of problems that commonly affect meta-analyses. Our results are largely robust against a number of issues that occasionally affect the reliability and validity of meta-analytic findings.

\section{DISCUSSION AND CONCLUSION}

As a discretionary set of activities that firms adopt, firms are likely to remain highly concerned with the question of whether, and if so how, CSP will help them to improve CFP. By articulating the four mediating pathways through which CSP may lead to higher CFP, our study provides evidence-based guidance on four directions that firms may take to implement CSP in a value-enhancing way: 1) by enhancing stakeholder endorsement, 2) by improving firm reputation, 3) by strengthening innovative capacity, and finally 4) by reducing firmspecific risk. Besides offering guidance to managerial decision-making, our theoretical framework also contributes to the existing literature on CSP and guides future research on the CSP-CFP relationship.

By theorizing a limited set of pathways that mediate the relationship between CSP and CFP, and by testing these mediating pathways using MASEM on the currently available body of empirical evidence, this study has developed a theoretical framework that can 
integrate an important part of the CSP-CFP literature. Being grounded in the foundational tenets of the CSR and stakeholder theory literatures, the framework developed in our study could be helpful in theoretically organizing the still growing and increasingly fine-grained body of research on the CSP-CFP relationship along the four mediating pathways it contains. More generally, our study demonstrates that synthesizing the available evidence in a field by first developing a theoretical framework that can explain the key relationships in that field, and by subsequently testing these key predictions using advanced meta-analytic techniques, may be a useful strategy to counter the empirically centrifugal and theoretically eclectic tendencies that may result from the use of sophisticated research designs (Bettis et al., 2014).

But while our theoretical framework comprising the four mediating pathways between CSP and CFP explains an important fraction of the total variation in CFP that is indirectly attributable to CSP, our results also show that a significant direct relationship between CSP and CFP remains after having accounted for the mediation effects theorized. This finding implies that there are additional mediating mechanisms connecting CSP with CFP that are not yet sufficiently understood, or that are currently under researched or even unobserved. An evident opportunity for future research therefore resides in the identification of previously undocumented mediating pathways connecting CSP with CFP.

More than ten years ago, Lockett and colleagues (2006) predicted that: 'the field of CSR will be characterized by a lack of convergence and, therefore, the body of CSR research will remain fragmented in terms of empirics, theory and (non) normative orientation' ( $p$ : 118). Based on the sheer number of studies included in our meta-analysis, it is clear that the empirical analysis of the CSP-CFP relationship has consistently been a key research focus of both the CSR and stakeholder theory literatures. But although our study suggests that empirical research converges along four pathways, Lockett and colleagues (2006) were at least partly right in their prediction that the field would continue to be theoretically fragmented. Due to the multiple theoretical perspectives that have inspired existing CSP studies, it currently still lacks a clear and dominant paradigm (Aguinis and Glavas, 2012; Gond and Crane, 2010) and seems to be in a 'continuing state of emergence' (Lockett et al., 2006: 133). By presenting an integrative theoretical framework, we aspire to help the field break out from this state of emergence. Because our framework is firmly rooted in the CSR and stakeholder theory literatures, rather than in any other 'borrowed' perspective, it has the potential to offer CSR and stakeholder theorizing an integrative framework that can avoid further fragmentation, and help the field progress in a more orchestrated manner.

\section{REFERENCES AVAILABLE FROM THE AUTHOR}

